\title{
3D Printed Flow Phantoms With Fiducial Markers for Super-Resolution Ultrasound Imaging
}

Ommen, Martin Lind; Schou, Mikkel; Zhang, Rujing; Villagómez Hoyos, Carlos Armando; Jensen, Jørgen Arendt; Larsen, Niels Bent; Thomsen, Erik Vilain

Published in:

2018 IEEE International Ultrasonics Symposium (IUS)

Link to article, DOI:

10.1109/ULTSYM.2018.8580217

Publication date:

2018

Document Version

Peer reviewed version

Link back to DTU Orbit

Citation (APA):

Ommen, M. L., Schou, M., Zhang, R., Villagómez Hoyos, C. A., Jensen, J. A., Larsen, N. B., \& Thomsen, E. V. (2018). 3D Printed Flow Phantoms With Fiducial Markers for Super-Resolution Ultrasound Imaging. In 2018 IEEE International Ultrasonics Symposium (IUS) IEEE. https://doi.org/10.1109/ULTSYM.2018.8580217

\section{General rights}

Copyright and moral rights for the publications made accessible in the public portal are retained by the authors and/or other copyright owners and it is a condition of accessing publications that users recognise and abide by the legal requirements associated with these rights.

- Users may download and print one copy of any publication from the public portal for the purpose of private study or research.

- You may not further distribute the material or use it for any profit-making activity or commercial gain

- You may freely distribute the URL identifying the publication in the public portal 


\title{
3D Printed Flow Phantoms with Fiducial Markers for Super-Resolution Ultrasound Imaging
}

\author{
$\underline{\text { Martin Lind Ommen }}^{1}$, Mikkel Schou ${ }^{2}$, Rujing Zhang ${ }^{1}$, Carlos Armando Villagómez Hoyos ${ }^{2}$, \\ Jørgen Arendt Jensen ${ }^{2}$, Niels Bent Larsen ${ }^{1}$, and Erik Vilain Thomsen ${ }^{1}$ \\ ${ }^{1}$ Department of Micro and Nanotechnology, Technical University of Denmark, Kgs. Lyngby, Denmark \\ ${ }^{2}$ Center for Fast Ultrasound Imaging, Technical University of Denmark, Kgs. Lyngby, Denmark
}

\begin{abstract}
The improved resolution provided by ultrasound super-resolution imaging (SRI) sets new demands on the fabrication of phantoms for the validation and verification of the technique. Phantoms should resemble tissue and replicate the 3D nature of tissue vasculature at the microvascular scale. This paper presents a potential method for creating complex 3D phantoms, via 3D printing of water-filled polymer networks. By using a custom-built stereolithographic printer, projected light of the desired patterns converts an aqueous poly(ethylene glycol) diacrylate (PEGDA) solution into a hydrogel, a material capable of containing $75 \mathrm{wt} \%$ of water. Due to the hydrogel mainly consisting of water, it will, from an acoustical point of view, respond very similar to tissue. A method for printing cavities as small as $(100 \mu \mathrm{m})^{3}$ is demonstrated, and a 3D printed flow phantom containing channels with cross sections of $(200 \mu \mathrm{m})^{2}$ is presented. The designed structures are geometrically manufactured with a $2 \%$ increase in dimensions. The potential for further reduction of the flow phantom channels size, makes 3D printing a promising method for obtaining microvascular-like structures.
\end{abstract}

Index Terms-3D printing, stereolithography, phantom, hydrogel, microvasculature, resolution, ultrasound

\section{INTRODUCTION}

Living tissue continuously adapts to changes in external stresses, or internal requirements. A local increase in cell activity will require an increase in nutrients and oxygen to the local tissue. One reason for increased cell activity could be cell proliferation due to cancer. The increased demands are met through increased vascularisation of the tissue [1]. This correlation between cancer and vascularisation can be used in the diagnosis of cancer patients. Once carcinogenic tissue has been detected, it will be possible to closely follow the vasculature response to any cancer treatments, with the potential to react fast to treatment responses.

Ultrasound super-resolution imaging (SRI) has within the last few years been introduced as a non-invasive method for imaging of the vasculature [2], [3]. The characteristic signal from contrast bubbles can be used to form images of higher resolution than would be possible due to the diffraction limit of regular ultrasound methods. To verify and optimise imaging- and tracking algorithms, it is necessary to use tissue mimicking phantoms, which not only recapitulate the acoustic properties of tissue, but also the scale and dimensionality of interest. The latter two points have proven difficult to engineer. At the introduction of the ultrasound SRI field, a few articles were published, demonstrating the method principles using measurements of phantoms. In one case, the channel dimensions were decreased to $40 \times 80 \mu \mathrm{m}^{2}$ [4] by defining the channel using UV lithography. However, in practice this imposes a limit of the channels being in a single plane. Other examples of phantoms consisting of tubes have been presented in [5] (3 mm diameter) and [6] (200 $\mu \mathrm{m}$ diameter). In both cases this is larger than the vessels of interest, which are less than $20 \mu \mathrm{m}$ in diameter.

$3 \mathrm{D}$ printing of polymers is a promising new technique, which allows the flexibility of fabricating complex 3D structures, as well as printing of very small features in the sub-100 $\mu \mathrm{m}$ range. Alignment of the ultrasound probe to the phantom becomes increasingly difficult as phantom features become smaller. Inclusion of fiducial markers within the phantoms can greatly reduce this problem.

We present a method for 3D printing of phantoms, by stereolithography. Our hypothesis is that by $3 \mathrm{D}$ printing phantoms using stereolithography, it is possible to create geometrically stable 3D phantoms to a precision within $5 \%$ of the designs, with acoustical properties similar to tissue, containing feature sizes relevant to $\mathrm{SRI}$ in the sub-100 $\mu \mathrm{m}$ range, print flow channels in 3D space and define fiducial markers with the purpose of probe alignment to the phantom features. Multiple iterations of fiducial markers have been made with a twofold purpose. Firstly, to investigate the type of markers made, and the influence on the contrast, and secondly, to investigate how small the markers can be made while still providing sufficient contrast.

\section{Materials AND Methods}

\section{A. Stereolithography}

Stereolithography uses a liquid resin hardened to the shape of the desired pattern through local illumination by a light source, in a layer by layer process to produce the designed $3 \mathrm{D}$ object. Fig. 1 illustrates the method employed. The printer vat contains the resin. The bottom of the vat is transparent to enable transmission of light to the resin. A glass slide is mounted on the fabrication stage, which is lowered into the resin, until a short distance from the vat bottom. The exact distance sets a limit on the thickness of the first printed layer. Upon illumination, the liquid resin will start crosslinking until reaching the 'gel point' at which the resin solidifies. The illumination system allows for local exposure of the polymer to enable printing of hollow structures. The fabrication stage is moved a specified distance away from the bottom of the vat once a layer has been exposed, thereby defining the next 


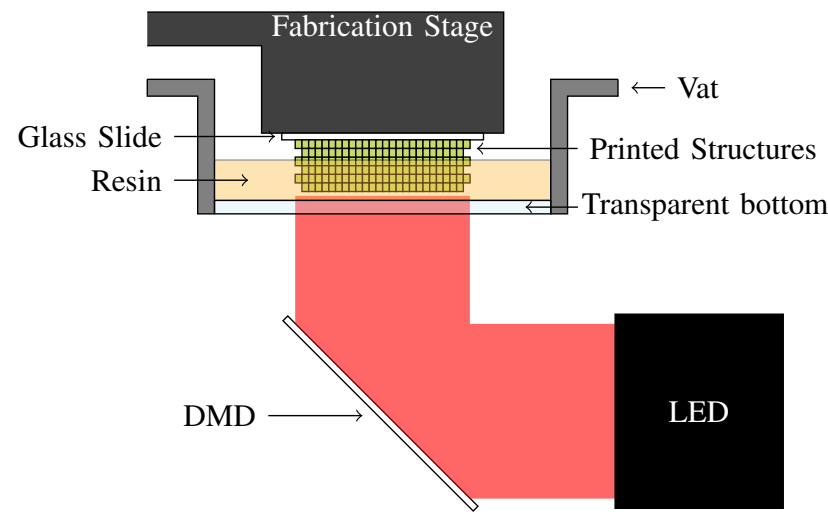

Figure 1. Sketch of the stereolithograhy setup. Light from an LED illuminates a digital mirror device (DMD), which reflects the light in the desired pattern through the transparent printer vat bottom. The illuminated resin in the vat will then start crosslinking. The initial layer of the printed structures is crosslinked to a glass slide mounted on the movable fabrication stage.

layer thickness. This is repeated until all layers of the object have been printed.

\section{B. Custom Built Stereolithographic Printer}

The printing setup has previously been described in [7]. Briefly, the custom-built high-resolution printer is based on a 1-to-1 projection of light reflected off a digital mirror device (DMD, $10.6 \mu \mathrm{m}$ pixel pitch, DLP9500UV, Texas Instruments; part of a V-9501 UV SuperSpeed Digital Light Processing module, Vialux) onto the transparent bottom of a vat containing the resin. The printing process is controlled by a custom written MATLAB (MathWorks) code, that synchronises digital mask exposure on the DMD, with light exposure using a $365 \mathrm{~nm}$ high power LED (LZ1-00UV00, Ledengin), and fabrication stage movement via a linear stage (LNR50S, Thor Labs). The power density at the vat bottom was $18 \mathrm{~mW} \mathrm{~cm}^{-2}$ as measured using a UV power meter $(\mathrm{S} 130 \mathrm{VC}+\mathrm{PM} 100 \mathrm{D}$, Thorlabs). The stage is moved in $20 \mu \mathrm{m}$ steps, matched to the light absorber concentration, which determines the vertical printing resolution. This results in an ultimate printing resolution of $10 \times 10 \times 20 \mu^{3}$.

\section{Resin}

The resin used for printing consists of 3 parts: an aqueous pre-polymer solution, a photo-initiator and a photo-absorber. The pre-polymer will polymerise to form a solid when locally initiated by the light activated photo-initiator. Light in a medium is attenuated according to Lambert-Beers law

$$
I=I_{0} e^{-\mu d},
$$

where $I_{0}$ is the initial intensity, $\mu$ is the attenuation coefficient, and $d$ is the depth at which the intensity, $I$, is measured. Fig. 2 shows two examples of the exponential decay of the light intensity in two different media with different $\mu$. The pre-polymer reaction initiated by the photo-initiator will need a certain dose (intensity times the exposure duration) of light to solidy (reach its gel point). Thus, illumination of the polymer will only crosslink the polymer until a certain depth. In Fig. 2 this is marked by $d_{1}$ and $d_{2}$ for the two

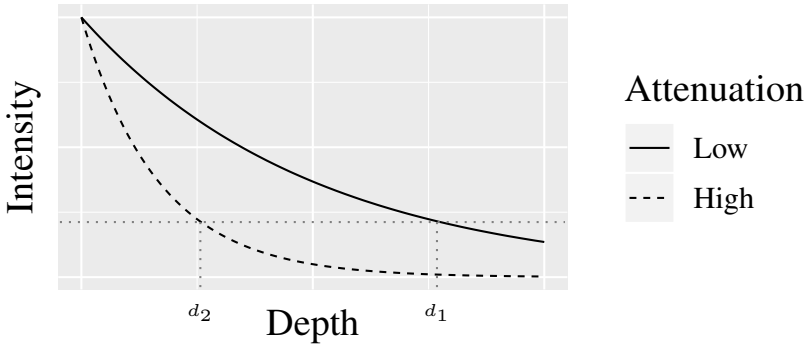

Figure 2. Light intensity against depth for two (arbitrary) different attenuation coefficients. $d_{1}$ and $d_{2}$ mark the depths, at which the light dose (intensity times exposure duration) has decreased to the threshold of resin solidification, marked by the grey horisontal dotted line.

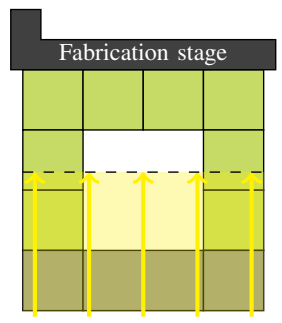

(a) Too little attenuation

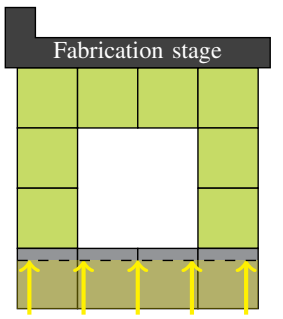

(b) Too much attenuation

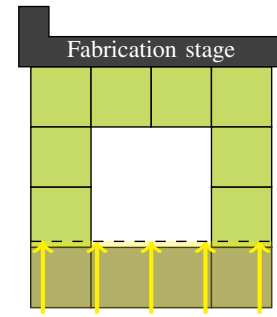

(c) Ideal attenuation
Figure 3. Sketches of the crosslinking depth for different levels of attenuation. The green squares are previously exposed voxels, and the grey squares are the voxels which are to be exposed in the new layer. The dashed lines mark the depth of the threshold dose. (a) has too little attenuation, and previous layers are are re-exposed. (b) has too much attenuation, and the newly exposed layer is unable to crosslink with the structures in the previous layer. (c) has sufficient attenuation, with only a minimum overlap in the exposed region to the previous crosslinked structures.

curves corresponding to different levels of attenuation. While the interlayer movement of the stage sets a lower limit on the vertical resolution, the light dose may be sufficiently large to induce further solidification in previously exposed layers, as illustrated in Fig. 3(a). The aqueous solution of prepolymer and photo-initiator has little attenuation, so light will propagate far into the resin before being absorbed. Addition of a highly absorbent photo-absorber allows to limit the propagation depth, thereby setting the depth resolution. A light absorber will modify $\mu$ of the solution according to (2),

$$
\mu=\epsilon c
$$

where $\epsilon$ is the extinction coefficient, and $c$ the concentration of the photo-absorber. Thus, $\mu$ can be modified by the type and concentration the added photo-absorber from a slowly attenuating medium such as water, exemplified by the solid curve and $d_{1}$ in Fig. 2, to a higher attenuating medium exemplified by the dashed curve and $d_{2}$. If $\mu$ becomes too large, the polymer will not crosslink sufficiently deep to chemically bond to the overlaying structures in the previous layer, as illustrated in Fig. 3(b) Thus, the choice of photo-absorber and concentration must be matched to get a slight overlap between the newly exposed regions, and the previously printed structures, as shown in Fig. 3(c)

The pre-polymer used is poly(ethylene glycol) diacrylate (PEGDA) $700 \mathrm{~g} / \mathrm{mol}$ at $200 \mathrm{mg} / \mathrm{mL}$. This polymer will contain $75 \mathrm{wt} \%$ water when converted to a hydrogel, thereby making it resemble tissue, both in terms of the water content [8] and, as a consequence, also in terms of the acousti- 


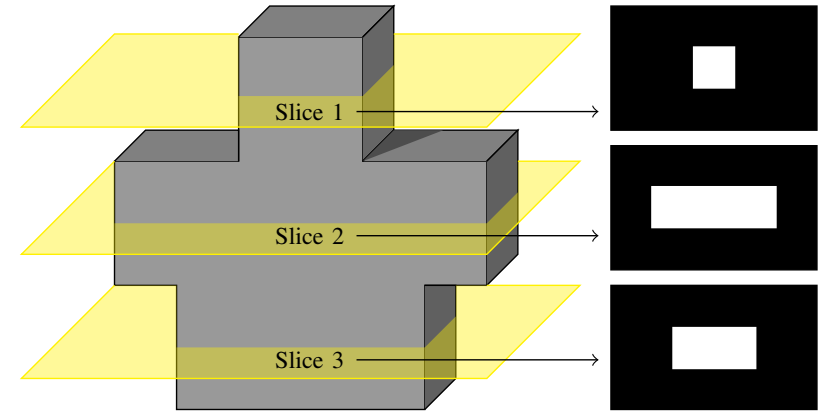

Figure 4. 3D model slicing. The slicing software takes a 3D model, as the one on the left, and decomposes it into slices, which are exported as .png files. The figures on the right are examples of slices corresponding to the yellow planes.

cal properties. The photo-initiator is lithium phenyl-2,4,6trimethylbenzoylphosphinate (LAP) at $5 \mathrm{mg} / \mathrm{mL}$, and is chosen for its water solubility and its absorption spectrum, which matches the light source used. The photo-absorber is quinoline yellow (QY) at a concentration of $12 \mathrm{mg} / \mathrm{mL}$, also chosen for its water solubility and high extinction coefficient at the wavelength used.

\section{3D Design Creation}

The design to be printed can be created in any 3D software. However, the 3D printer MATLAB code requires that the 3D model is sliced into separate layers. This can be done by the open source slicing software Slic3r (www.slic3r.org), which converts the model to a set of portable network graphics (.png) files. Additionally, the slicing software produces a build list, detailing the order and exposure time of each individual layer. Fig. 4 shows the slicing concept, where a 3D model, on the left, is sliced into separate .pngs on the right, at each of the yellow planes.

When using the slicing software, the exact vertical placement of the slice is not controlled. The features of the $3 \mathrm{D}$ model may end up being split into multiple layers, if they do not align precisely with the slices. On the other hand, if the designs are simple, the .png files and the build list can be made manually, giving full control of the individual slices. As structures become smaller, this level of control will become increasingly more important. For the investigation of fiducial marker creation, all models have been made manually, by defining the individual t.pngs in a MATLAB script.

\section{E. Fiducial Marker Designs}

Two types of markers were tested: hollow markers and solid markers. Ultrasound scattering is a function of perturbations in density and propagation velocity [9]. At the given polymer crosslinking density, the speed of sound within the material is practically constant. However, as more polymer polymerises, the crosslinking density will increase, which in turn increases scattering. Thus, when hollow markers are made, the scattering will increase as the density changes from that of the polymer, to that of the water contained within the hollow marker. When solid markers are made, the scattering increases due to a local increase in the polymer density, formed by overexposure of that region.

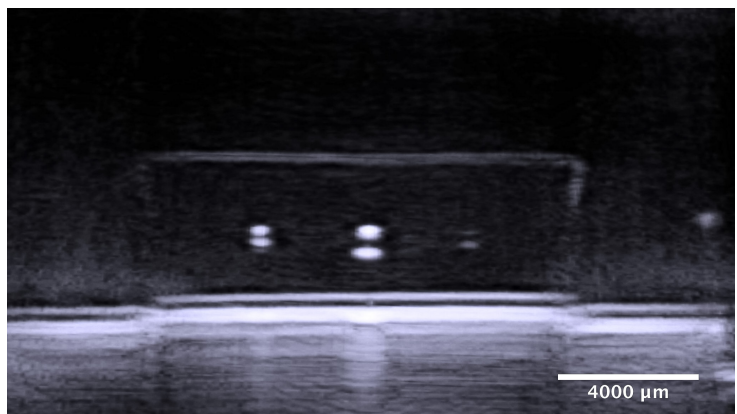

Figure 5. Ultrasound image showing the signal of two types of fiducial markers: Hollow (left and centre) and solid (right). The hollow markers have significantly larger contrast than the solid markers. Each marker shows two contrast regions. These correspond to the top and bottom of the cavity or solid region.

\section{RESULTS}

\section{A. Type of Markers}

A 3D printed phantom containing both solid and hollow markers was made to investigate the difference in contrast from the two types of markers. All ultrasound images presented have been scanned using a BK Medical "Hockey Stick" X18L5s probe and a BK 5000 scanner. A cross-sectional image showing both types of markers can be seen in Fig. 5 The left and centre markers are hollow markers, and the right marker is a solid marker. The left and right markers are both $(240 \mu \mathrm{m})^{3}$, and the centre marker is $(400 \mu \mathrm{m})^{3}$. Each marker can be seen as two high contrast spots on top of each other. The reason is that the propagation media density changes twice for each marker, namely at the top of the marker (the transition from polymer to unexposed resin - or higher density polymer for the solid markers), and at the bottom of the marker (back to the polymer from the unexposed polymer solution - or high-density polymer for the solid markers). Each of these interfaces will result in scattering of sound, and therefore contrast in the image. The markers on the left and the right are of the same size, and therefore suitable for comparison. The reflected intensity from the solid marker is measured to be $28 \mathrm{~dB}$ lower than the reflected intensity from the hollow marker.

\section{B. Size of Markers}

In an iterative process, the size of the markers was sought optimised. Fig. 6 shows a cross-sectional image of a single line of markers of decreasing sizes from $(140 \mu \mathrm{m})^{3}$ on the far right and decreasing by $10 \mu \mathrm{m}$ to $(60 \mu \mathrm{m})^{3}$ on the far left, for a total of 9 markers across the phantom. Five markers are clearly visible and useful as point spread functions, with the smallest being $(100 \mu \mathrm{m})^{3}$. Arguably, a few more of the smaller markers can be seen, barely above the noise level. The reason may be the limit of the chosen design method, but it could also be probe alignment, the choice of focus point, or a sub-optimal time gain compensation.

\section{Manufacturing Accuracy}

The polymer networks, and thereby the phantoms, will swell in size after printing due to water uptake. This means that 


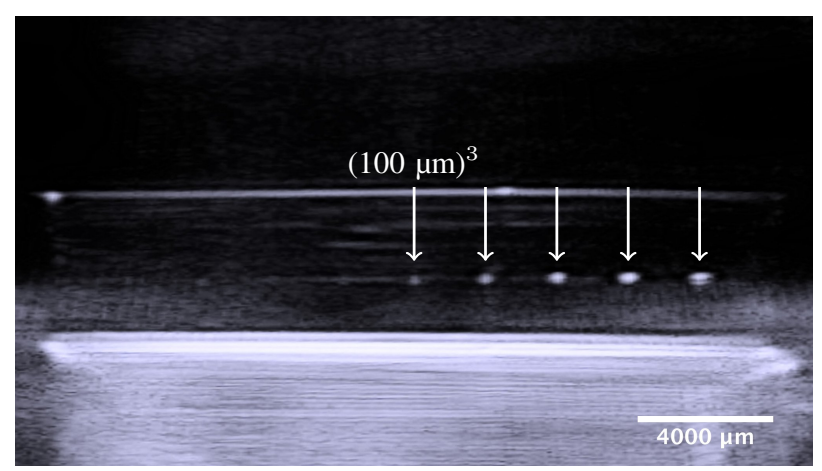

Figure 6. Ultrasound image showing the signal from hollow markers of decreasing design sizes from $(140 \mu \mathrm{m})^{3}$ on the far right and decreasing by $10 \mu \mathrm{m}$ in all dimensions to $(60 \mu \mathrm{m})^{3}$ on the far left. The clearly visible markers are indicated by arrows.

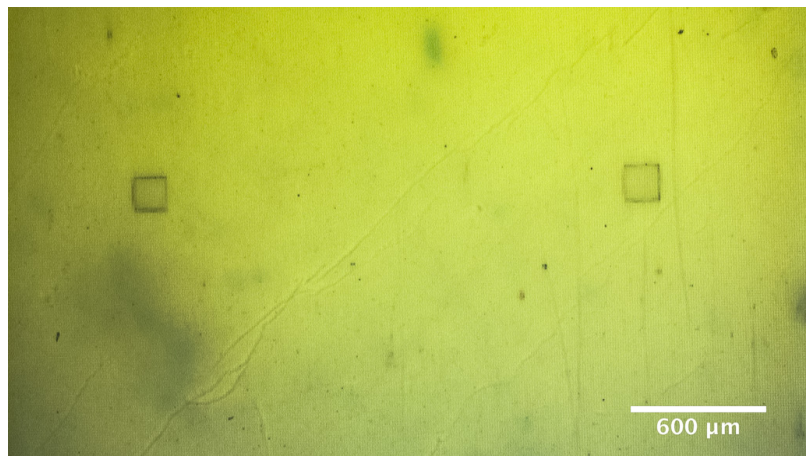

Figure 7. Optical microscope image of two square fiducial markers.

the designed structures will need to account for this, by incorporating a scaling factor corresponding to the increase. This difference is quantified by optical microscope- and ultrasound images. Fig. 7 shows an optical microscope image of two hollow fiducial markers. The designed pitch between all markers was $2074 \mu \mathrm{m}$. By measuring the pitch in optical microscope images similar to Fig. 7, the distance was found to be $2115 \pm 9 \mu \mathrm{m}$ (an average deviation from the design of $2 \%$ ). In a similar investigation of the ultrasound image in Fig. 6, the pitch was found to be $2078 \pm 71 \mu \mathrm{m}$ (an average deviation of $0.2 \%$ ).

\section{Flow Channel Phantom}

Fig. 8 shows an ultrasound SRI image of micro-bubble flow through a channel with a cross section of $(200 \mu \mathrm{m})^{2}$ of a 3D printed phantom without fiducial markers. Liquid enters the lower channel to the right and leaves the upper channel to the right. The inserted colour wheel indicates the flow direction of the micro-bubbles. Fewer detected bubbles are observed passing through the top channel. Possible reasons could be channel misalignment or that the contrast bubbles were destroyed before reaching the end of the channel. Fig. 8 demonstrates that the channel is perfusable, and that the bubbles flow from the entrance in the lower right, up into the top channel, and out through the top right, as expected. This preliminary flow phantom demonstrates the 3D capabilities of printing by stereolithography. Using the same printing setup, channels with a cross section of $(100 \mu \mathrm{m})^{2}$ have been demonstrated for other purposes than ultrasound [7],
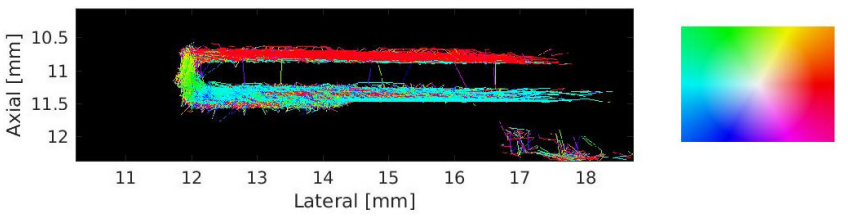

Figure 8. Super-resolution image of micro-bubbles through a 3D printed channel. The colour wheel indicates the micro-bubble flow direction.

and in-house testing have demonstrated $(50 \mu \mathrm{m})^{2}$ perfusable channels.

\section{DISCUSSION AND CONCLUSION}

We have presented a custom-built stereolithographic 3D printer capable of printing hydrogels, which are capable of storing $75 \mathrm{wt} \%$ of water, thereby resembling tissue in terms of the acoustic properties, making them well suited for the fabrication of ultrasound phantoms.

Fiducial markers have been printed, with hollow markers providing $28 \mathrm{~dB}$ more signal than solid markers. Markers of only $(100 \mu \mathrm{m})^{3}$ have been shown to provide a well defined point spread function. A 3D printed flow phantom has been demonstrated with $(200 \mu \mathrm{m})^{2}$ cross sectional channels. The designed structures have been printed with a final $2 \%$ increase in dimensions.

The preliminary findings presented within the previous sections, highlight the potential for stereolithographic printing of phantoms, and furthermore indicates that the fiducial markers obtained in this work are not demonstrating the ultimate resolution limit. Optimised printing schemes will be explored in an effort to obtain the highest possible resolution of the printing system.

\section{REFERENCES}

[1] D. Hanahan and R. A. Weinberg. Hallmarks of cancer: The next generation. Cell, 144(5):646-674, 2011.

[2] C. Errico, J. Pierre, S. Pezet, Y. Desailly, Z. Lenkei, O. Couture, and M. Tanter. Ultrafast ultrasound localization microscopy for deep superresolution vascular imaging. Nature, 527(7579):499-+, 2015.

[3] K. Christensen-Jeffries, R. J. Browning, M. Tang, C. Dunsby, and R. J. Eckersley. In vivo acoustic super-resolution and super-resolved velocity mapping using microbubbles. IEEE Trans. Med. Imag., 34(2):433-440, 2015 .

[4] Y. Desailly, O. Couture, M. Fink, and M. Tanter. Sono-activated ultrasound localization microscopy. Appl. Phys. Lett., 103(17):174107, 2013.

[5] O. M. Viessmann, R. J. Eckersley, K. Christensen-Jeffries, M. X. Tang, and C. Dunsby. Acoustic super-resolution with ultrasound and microbubbles. Phys. Med. Biol., 58(18):6447-6458, 2013.

[6] K. Christensen-Jeffries, J. Brown, P. Aljabar, M. Tang, C. Dunsby, and R. J. Eckersley. 3-D in vitro acoustic super-resolution and superresolved velocity mapping using microbubbles. IEEE Trans. Ultrason., Ferroelectr., Freq. Control, 64(10):1478-1486, 2017.

[7] R. Zhang and N. B. Larsen. Stereolithographic hydrogel printing of 3D culture chips with biofunctionalized complex 3D perfusion networks. $\mathrm{Lab}$ Chip, 17(24):4273-4282, 2017.

[8] R. M. Forbes, A. R. Cooper, and H. H. Mitchell. The composition of the adult human body as determined by chemical analysis. J. Biol. Chem., 203(1):359-366, 1953.

[9] J. A. Jensen. A model for the propagation and scattering of ultrasound in tissue. J. Acoust. Soc. Am., 89(1):182-190, 1991 